\title{
Influence of Nanosized Silicon Oxide on the Luminescent Properties of ZnO Nanoparticles
}

\author{
Vitaliy Shvalagin, ${ }^{1}$ Galyna Grodziuk, ${ }^{1}$ Olha Sarapulova, ${ }^{2}$ Misha Kurmach, ${ }^{1}$ \\ Vasyl Granchak, ${ }^{1}$ and Valentyn Sherstiuk ${ }^{2}$ \\ ${ }^{1}$ L.V. Pisarzhevsky Institute of Physical Chemistry, National Academy of Science of Ukraine, 31 Nauky Avenue, Kyiv 03028, Ukraine \\ ${ }^{2}$ Institute of Publishing and Printing, National Technical University of Ukraine "Kyiv Polytechnic Institute", 1/37 Yangel Street, \\ Kyiv 03056, Ukraine
}

Correspondence should be addressed to Olha Sarapulova; olhasarapulova@gmail.com

Received 26 January 2016; Revised 30 March 2016; Accepted 3 April 2016

Academic Editor: Kaibo Zheng

Copyright (C) 2016 Vitaliy Shvalagin et al. This is an open access article distributed under the Creative Commons Attribution License, which permits unrestricted use, distribution, and reproduction in any medium, provided the original work is properly cited.

\begin{abstract}
For practical use of nanosized zinc oxide as the phosphor its luminescence quantum yields should be maximized. The aim of this work was to enhance luminescent properties of $\mathrm{ZnO}$ nanoparticles and obtain high-luminescent $\mathrm{ZnO} / \mathrm{SiO}_{2}$ composites using simpler approaches to colloidal synthesis. The luminescence intensity of zinc oxide nanoparticles was increased about 3 times by addition of silica nanocrystals to the source solutions during the synthesis of $\mathrm{ZnO}$ nanoparticles. Then the quantum yield of luminescence of the obtained $\mathrm{ZnO} / \mathrm{SiO}_{2}$ composites is more than $30 \%$. Such an impact of silica is suggested to be caused by the distribution of $\mathrm{ZnO}$ nanocrystals on the surface of silica, which reduces the probability of separation of photogenerated charges between the zinc oxide nanoparticles of different sizes, and as a consequence, there is a significant increase of the luminescence intensity of $\mathrm{ZnO}$ nanoparticles. This way of increasing nano- $\mathrm{ZnO}$ luminescence intensity facilitates its use in a variety of devices, including optical ultraviolet and visible screens, luminescent markers, antibacterial coatings, luminescent solar concentrators, luminescent inks for security printing, and food packaging with abilities of informing consumers about the quality and safety of the packaged product.
\end{abstract}

\section{Introduction}

Semiconductor nanoparticles are being more intensively studied in recent years as promising objects of new functional materials with predetermined properties, due to the presence of a number of specific properties that significantly distinguish them from the bulk material of the same chemical composition [1]. These materials may find use in a variety of devices, including optical ultraviolet and visible screens, luminescent markers, antibacterial coatings, luminescent solar concentrators, luminescent inks for security printing, and food packaging with abilities of informing consumers about the quality and safety of consumption of the packaged product. One of the materials that can be used in most of the mentioned above devices is zinc oxide of nanoscale dispersion, due to intense absorption band in the ultraviolet region, antibacterial properties, and intensive luminescence in a wide range of the visible spectrum [2-4]. Among the additional advantages of nano- $\mathrm{ZnO}$, compared with a number of luminescent materials, there are nontoxicity, low cost, and ease of producing nanoparticles of a given size in a range of organic solvents.

However, it should be noted that for practical use of nanosized zinc oxide as the phosphor its luminescence quantum yields should be maximized. One approach to this would be introduction of nano-zinc oxide into silicon oxide matrix, which can significantly affect the luminescent properties of $\mathrm{ZnO}$ nanoparticles [5-7]. However, in most of such cases, the luminescence quantum yield of zinc oxide remains low (less than 10\%) $[8,9]$. To obtain higher quantum yield of luminescence of such composites, their synthesis process involved many steps and required long-term postsynthesis treatment [10]. 
In this context, the aim of this work was to obtain highluminescent materials based on zinc oxide nanoparticles modified by nanosized $\mathrm{SiO}_{2}$, using simpler approaches to colloidal synthesis.

\section{Materials and Methods}

$\mathrm{Zn}\left(\mathrm{CH}_{3} \mathrm{COO}\right)_{2}, \mathrm{NaOH}$, category "chemical purity," and hydrophobic silica of Evonik production were used. As the medium absolute ethanol was used, obtained by boiling it with heated calcium oxide, followed by distilling according to the method, described in [11]. Optical density of the suspensions was measured with a spectrophotometer Analytik Jena Specord 210. Photoluminescence spectra (PL) and luminescence excitation spectra were recorded with a luminescent spectrometer Perkin-Elmer LS55 when excited by light with the wavelength $\lambda_{\text {exc. }}=340 \mathrm{~nm}$ and at the luminescence maximum at $\lambda_{\text {emission }}=540 \mathrm{~nm}$, respectively. Hydrodynamic particle size was determined by laser photocorrelation spectroscopy using a Malvern ZetaSizer Nano $\mathrm{S}$. The XRD spectra were registered on Bruker D8 Advance diffractometer with the $\mathrm{Cu} \mathrm{K} \alpha$ irradiation.

$\mathrm{ZnO}$ nanoparticles were prepared according to known methods [12-14] by alkaline hydrolysis of zinc acetate under the influence of sodium hydroxide in absolute ethanol. Thoroughly triturated anhydrous samples of $\mathrm{Zn}\left(\mathrm{CH}_{3} \mathrm{COO}\right)_{2}$ and $\mathrm{NaOH}$ were dissolved in absolute ethanol. The acetate and alkali solutions were cooled to $0^{\circ} \mathrm{C}$ and slowly mixed with vigorous stirring by dropwise addition of $\mathrm{NaOH}$ solution to $\mathrm{Zn}\left(\mathrm{CH}_{3} \mathrm{COO}\right)_{2}$ solution. After mixing the reactants, the colloids were maintained at $60^{\circ} \mathrm{C}$ for two hours. The average size of $\mathrm{ZnO}$ nanoparticles was altered by reducing the concentration of precursors from $2 \times 10^{-2}$ to $2 \times 10^{-3} \mathrm{M}$.

$\mathrm{ZnO} / \mathrm{SiO}_{2}$ composites were obtained in two ways. In the first case, the required amount of hydrophobic silica was added to already formed $\mathrm{ZnO}$ nanoparticles and stirred vigorously on a magnetic stirrer until a homogeneous dispersion was obtained. In the second case, a sample of silica was dispersed in ethanol solution immediately after dissolving $\mathrm{Zn}\left(\mathrm{CH}_{3} \mathrm{COO}\right)_{2}$. Then the ethanol solutions of zinc acetate with $\mathrm{SiO}_{2}$ dispersion and the $\mathrm{NaOH}$ solution were cooled to $0^{\circ} \mathrm{C}$ and slowly mixed with vigorous stirring, as in the previous case. After mixing the reagents, the colloids were also maintained at $60^{\circ} \mathrm{C}$ for two hours.

The photoluminescence quantum yield of the obtained $\mathrm{ZnO} / \mathrm{SiO}_{2}$ composites was calculated using a solid anthracene as a standard with $100 \%$ quantum yield.

\section{Results and Discussion}

The absorption spectrum of the obtained zinc oxide nanoparticles with a concentration of $2 \times 10^{-2} \mathrm{M}$ in ethanol (Figure 1 , curve 1) contains an intense band with a long-wavelength edge at $355 \mathrm{~nm}$, which corresponds to the band gap $\left(E_{g}\right)$ of $\mathrm{ZnO}$ nanoparticles $3.5 \mathrm{eV}$. According to the calibration curve of $E_{g}$ of $\mathrm{ZnO}$ nanoparticles dependency on their size [14], the value of $E_{g}$ is characteristic of the medium size of zinc oxide nanoparticles of about $4.4 \mathrm{~nm}$.

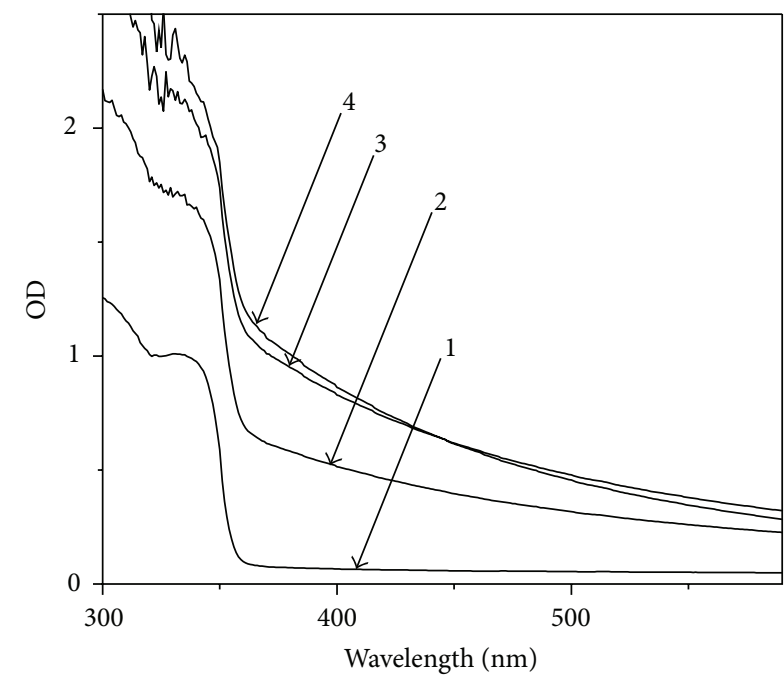

FIgURE 1: The absorption spectra of colloidal solutions of $\mathrm{ZnO}$ nanoparticles without $\mathrm{SiO}_{2}$ (1) and in the presence of $0.25 \mathrm{~g}(2)$, $0.625 \mathrm{~g}$ (3), and $1.25 \mathrm{~g}$ of silica (4) in $10 \mathrm{~mL}$ of zinc oxide solution. $[\mathrm{ZnO}]=2 \times 10^{-2} \mathrm{~mol} / \mathrm{L}, l=0.1 \mathrm{~cm}$.

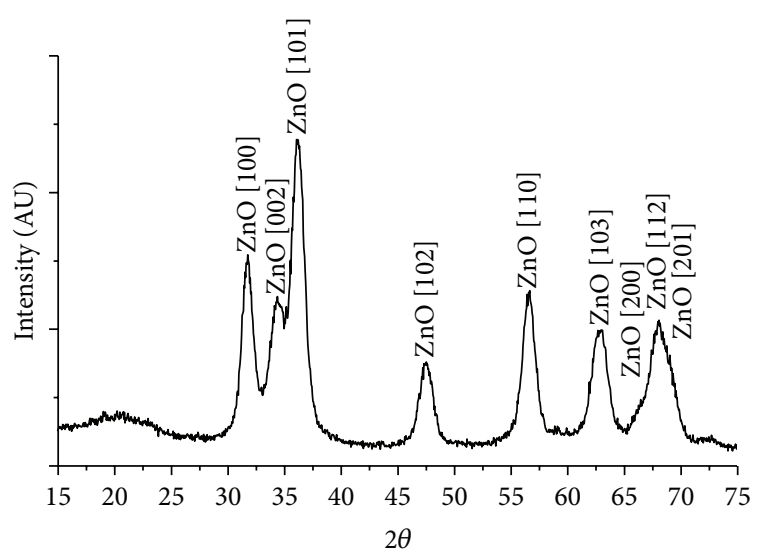

FIGURE 2: $\mathrm{XRD}$ results for $\mathrm{ZnO}$ nanoparticles.

In order to confirm the formation of zinc oxide, the results of XRD data are shown in Figure 2. In the diffraction pattern, presented in Figure 2, there are only peaks which are typical for hexagonal $\mathrm{ZnO}$ [15]. Broadening the spectral reflections indicates that the sample is composed of ultrafine particles of $\mathrm{ZnO}$.

It has been found that by adding $\mathrm{SiO}_{2}$ to clear ethanol solution of colloidal zinc oxide nanoparticles after their synthesis leads to an increase of the optical density of such dispersions in the entire investigated range (Figure 1, curves $2-4)$. Given that the $\mathrm{SiO}_{2}$ does not absorb light in this range, it can be assumed that the described changes in the optical characteristics of the solution take place due to the scattering of light by silica particles, which increases with the increase of the amount of silica in the colloidal solution.

According to the passport data of $\mathrm{SiO}_{2}$, nanoparticles used in the study have a size of about 15-20 nm. However, the data obtained by the method of dynamic light scattering show 


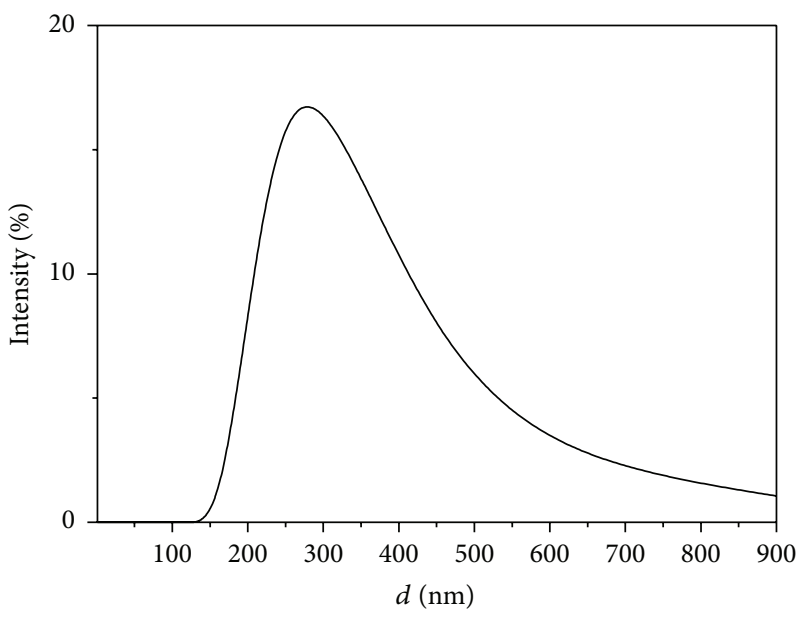

Figure 3: The curve of $\mathrm{SiO}_{2}$ nanoparticles distribution in size in absolute ethanol (according to dynamic light scattering spectroscopy).

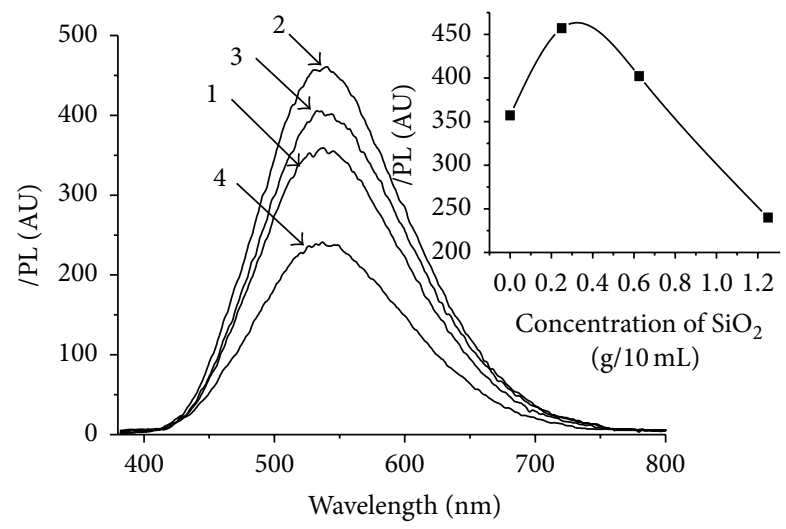

FIGURE 4: The luminescence spectra of the colloidal solutions of $\mathrm{ZnO}$ nanoparticles without $\mathrm{SiO}_{2}(1)$ and in the presence of $0.25 \mathrm{~g} \mathrm{(2)}$, $0.625 \mathrm{~g} \mathrm{(3)}$, and $1.25 \mathrm{~g}$ of silica (4) in $10 \mathrm{~mL}$ of zinc oxide solution. $[\mathrm{ZnO}]=2 \times 10^{-2} \mathrm{~mol} / \mathrm{L}, \lambda_{\text {exc. }}=340 \mathrm{~nm}$. The inset shows the dependency of luminescence intensity of $\mathrm{ZnO}$ nanoparticles on the amount of $\mathrm{SiO}_{2}$ introduced into the solution. $\lambda_{\text {emission }}=540 \mathrm{~nm}$.

that $\mathrm{SiO}_{2}$ nanoparticles used in the study are in alcoholic solutions in the form of aggregates with an average size of about $300 \mathrm{~nm}$ (Figure 3). The formation of such units, in authors' opinion, leads to such a significant scattering of light in this case.

The dependence of the luminescence intensity of $\mathrm{ZnO}$ nanoparticle colloids on the content of silica looks like a curve (Figure 4) with the maximum in the region of $0.2-0.4 \mathrm{~g} \mathrm{SiO}_{2}$ per $10 \mathrm{~mL}$ of $\mathrm{ZnO}$ nanoparticle colloid.

The increase of the luminescence intensity of zinc oxide nanoparticles with a relatively low content of $\mathrm{SiO}_{2}$ can be explained on the basis of the electronic properties of the semiconductor particles. As shown in the first paragraph of this section, the average size of zinc oxide nanoparticles obtained in the study was about $4.4 \mathrm{~nm}$. For such $\mathrm{ZnO}$ particle size quantum size effects are characteristic, such as changing the position of the semiconductor flat band when it is resized [14].

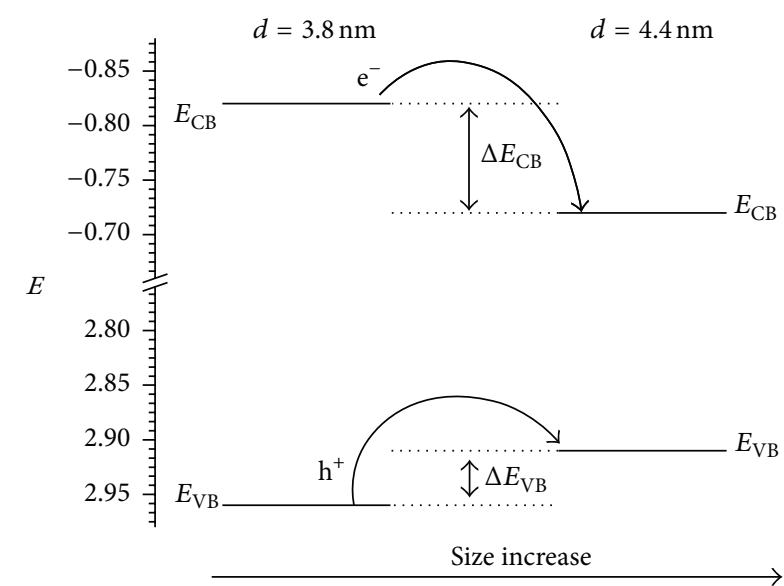

FIgURE 5: The energy diagram of $\mathrm{ZnO}$ nanoparticles of two different sizes.

As the obtained zinc oxide nanoparticles are characterized by size distribution, the position of their conduction and valence bands area differs depending on the specific particle size. Given the significant difference between the values of the effective masses of the charge carriers in zinc oxide $m_{e}^{*}=$ $0.26, m_{h}^{*}=0.59[12,16]$ and the known expressions for calculating the potential of the conduction band $\left(E_{\mathrm{CB}}\right)$ and valence band $\left(E_{\mathrm{VB}}\right)$ of semiconductor nanoparticles $[1,17]$, it can be shown that the energy gap between the positions of conduction bands of nanoparticles of different sizes $\left(E_{\mathrm{CB}}\right)$ is substantially bigger than the energy gap between their valence bands $\left(E_{\mathrm{VB}}\right)$. Visually such a difference is shown in the energy diagram of $\mathrm{ZnO}$ nanoparticles of two different sizes (Figure 5). Such differences in the energy positions of allowed bands of a semiconductor, according to [1], will lead to a more efficient transport of a photogenerated electron from the conduction band of the nanoparticles with smaller size into the conduction band of larger nanoparticles, in comparison with the transfer efficiency of photogenerated holes between their valence bands. These processes will facilitate the separation of photogenerated charges between the phosphor nanoparticles and, consequently, the reduction of the efficiency of $\mathrm{ZnO}$ nanoparticles luminescence. Since the adsorption of zinc oxide nanoparticles on the surface of $\mathrm{SiO}_{2}$ may limit direct contact between the semiconductor nanoparticles and thus limit the separation of photogenerated charges in them, it will help increase their luminescence intensity. This phenomenon is observed experimentally.

For additional verification of the assumption about luminescence quenching, the authors obtained zinc oxide nanoparticles of two different sizes according to the procedure described in [14] by varying $\mathrm{ZnO}$ concentration in the synthesis from $2 \times 10^{-2}$ to $2 \times 10^{-3} \mathrm{M}$. Thus, as can be seen from the absorption spectra of the solutions, represented in Figure 6, curves 1 and 2, respectively, the absorption edge of zinc oxide nanoparticles is shifted from 356 to $345 \mathrm{~nm}$, which corresponds to a reduction of crystal size from 4.4 to $3.8 \mathrm{~nm}$ [14]. After that, colloids containing such particles were mixed in different ratios and stirred at $60^{\circ} \mathrm{C}$ for $10 \mathrm{~min}$. It has 


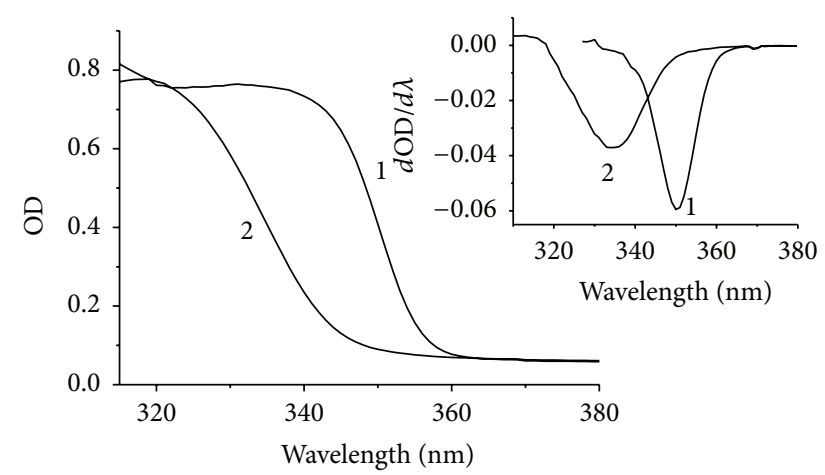

Figure 6: The absorption spectra of colloidal solutions of $\mathrm{ZnO}$ nanoparticles in ethanol with zinc oxide concentration during the synthesis: $[\mathrm{ZnO}]=2 \times 10^{-2} \mathrm{~mol} / \mathrm{L}(1) ;[\mathrm{ZnO}]=2 \times 10^{-3} \mathrm{~mol} / \mathrm{L}(2)$. The inset shows the first derivative of the absorption curves 1 and 2 , respectively. The optical thickness of the cuvette $l=1.0 \mathrm{~cm}$; the concentration during the spectra recording $[\mathrm{ZnO}]=2 \times 10^{-3} \mathrm{~mol} / \mathrm{L}$.

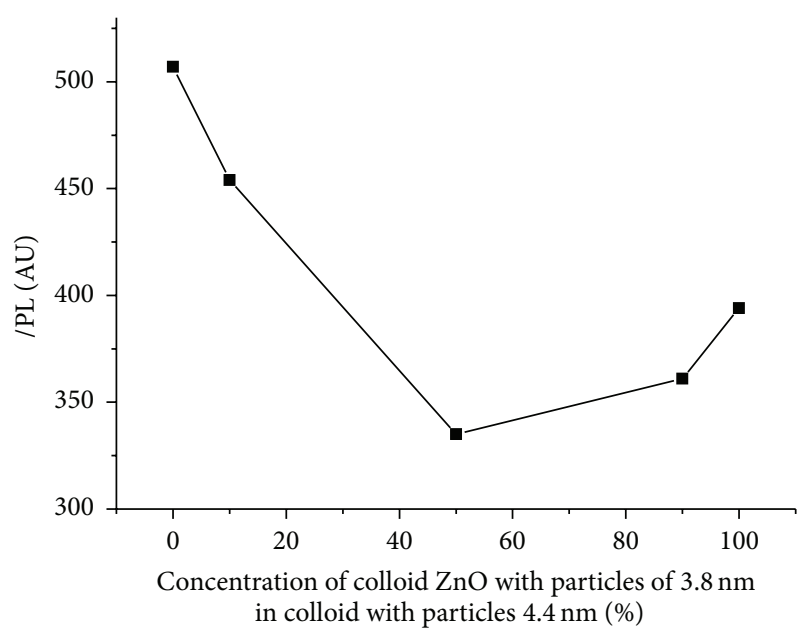

FIgURE 7: The dependence of the luminescence intensity of the colloid $\mathrm{ZnO}$ nanoparticles with size of $4.4 \mathrm{~nm}$ on the content of the colloid $\mathrm{ZnO}$ particles of $3.8 \mathrm{~nm}$. $[\mathrm{ZnO}]=2 \times 10^{-3} \mathrm{~mol} / \mathrm{L}$.

been found that the curve of the dependency of luminescence intensity of the colloid of $\mathrm{ZnO}$ nanoparticles with the size of $4.4 \mathrm{~nm}$ on their content of the colloid of particles with smaller size $(3.8 \mathrm{~nm})$ has the form of a curve with a minimum (Figure 7), in which luminescence intensity of the mixture is significantly lower than the luminescence intensity of the initial $\mathrm{ZnO}$ nanoparticle colloids. These results support the hypothesis about charge separation between nanoparticles of different sizes and the resulting luminescence intensity decrease.

The substantially less luminescence intensity obtained in the nanoparticles of $3.8 \mathrm{~nm}$ in comparison with the particle size of $4.4 \mathrm{~nm}$ (Figure 7, utmost points on the curve) can serve as further confirmation of the influence of the spatial separation of photogenerated charges between the semiconductor particles in contact on their luminescent properties. As can be seen from Figure 6, inset, the width of the first derivative of the absorption spectrum of a nanoparticle size of $3.8 \mathrm{~nm}$

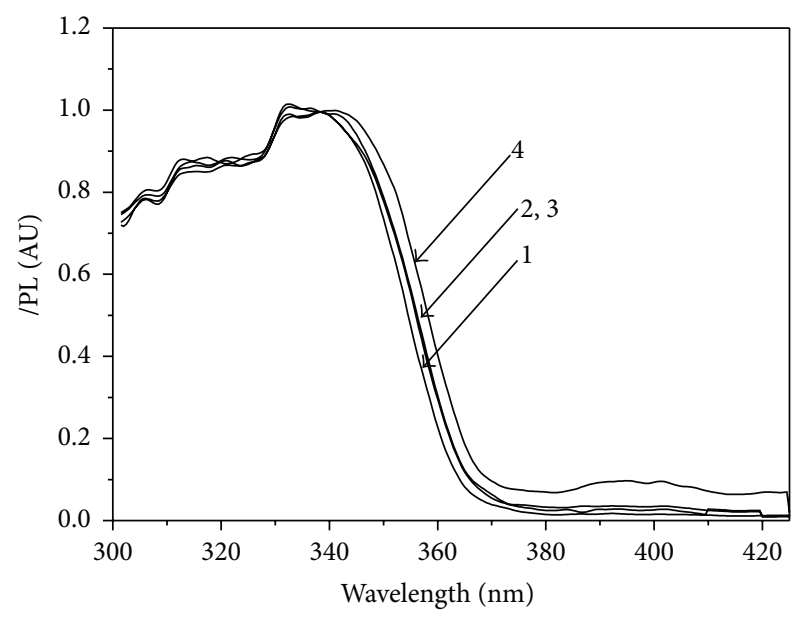

FIGURE 8: Normalized luminescence excitation spectra of the colloidal solutions of $\mathrm{ZnO}$ nanoparticles without $\mathrm{SiO}_{2}(1)$ and in the presence of $0.25 \mathrm{~g}(2), 0.625 \mathrm{~g}(3)$, and $1.25 \mathrm{~g}$ of silica (4) in $10 \mathrm{~mL}$ of zinc oxide solution. $[\mathrm{ZnO}]=2 \times 10^{-2} \mathrm{~mol} / \mathrm{L} ; \lambda_{\text {registration }}=540 \mathrm{~nm}$.

(Figure 6, inset, curve 2) is greater in comparison with the width of derivative for the particle size of $4.4 \mathrm{~nm}$ (Figure 6, inset, curve 1). This may be due to broader distribution of $\mathrm{ZnO}$ nanoparticles with a mean of $3.8 \mathrm{~nm}$ in size [18]. These differences, according to the authors' hypothesis, should lead to the improved spatial separation of photogenerated charges between the semiconductor particles with a larger size distribution and consequently to reduction of their luminescence intensity compared with the particles with a smaller size distribution. This phenomenon is observed experimentally.

At a high content of $\mathrm{SiO}_{2}$ (Figure 4) there is a decrease in the intensity of luminescence of zinc oxide nanoparticles, which may be due to the substantial light scattering at high content of silica particles and a nonuniform distribution of zinc oxide nanoparticles on the surface of the $\mathrm{SiO}_{2}$ crystals.

It should be noted that there are no significant changes in the location of the long-wave absorption edge of zinc oxide nanoparticles and its shape in the absorption spectra (Figure 1) and the luminescence excitation spectra (Figure 8) of $\mathrm{ZnO} / \mathrm{SiO}_{2}$ nanoparticles, which may indicate the absence of influence of $\mathrm{SiO}_{2}$ on the size and energy characteristics of zinc oxide nanoparticles.

Since the addition of silica nanoparticles into colloidal solutions of $\mathrm{ZnO}$ nanocrystals allows increasing their luminescence intensity, the authors decided to investigate this effect of $\mathrm{SiO}_{2}$ when administered directly during the synthesis of zinc oxide nanoparticles. In particular, it was found that after the introduction of $\mathrm{SiO}_{2}$ during the synthesis of zinc oxide nanoparticles and colloid aging by a standard technique, in addition to the absorption spectrum containing the band of colloidal $\mathrm{ZnO}$ in the range of $\lambda \leq 350-$ $360 \mathrm{~nm}$ (Figure 9, curve 1), there is an additional increase in optical density in the investigated range (Figure 9, curves 2-6), which, similarly to the previous case, is caused by the scattering of light by silicon oxide particles. 


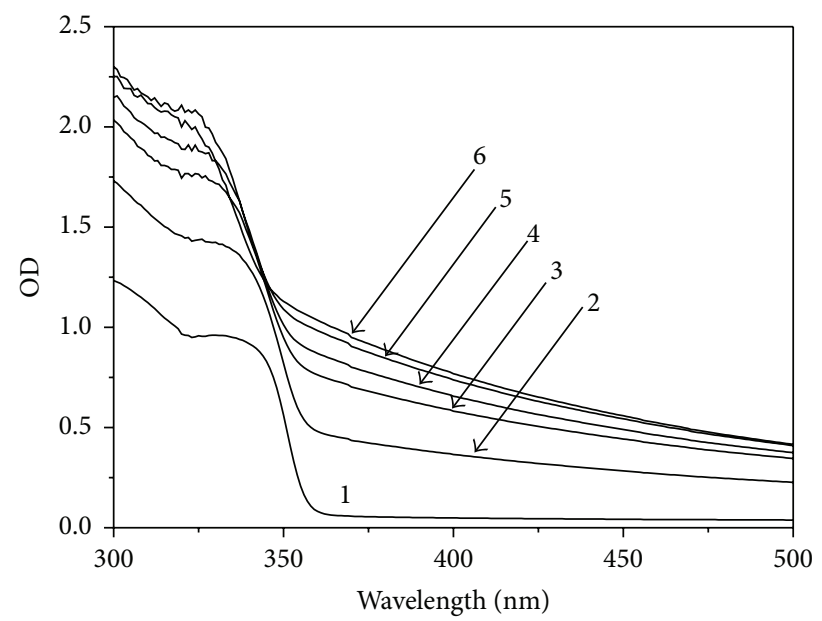

Figure 9: The absorption spectra of the colloidal solutions of $\mathrm{ZnO}$ nanoparticles without $\mathrm{SiO}_{2}(1)$ and with provisionally adding $0.2 \mathrm{~g}$ (2), $0.4 \mathrm{~g} \mathrm{(3),} 0.6 \mathrm{~g}(4), 0.8 \mathrm{~g}$ (5), and $1 \mathrm{~g}$ of silica (6) to $10 \mathrm{~mL}$ of zinc oxide solution. $[\mathrm{ZnO}]=2 \times 10^{-2} \mathrm{~mol} / \mathrm{L} ; l=0.1 \mathrm{~cm}$.

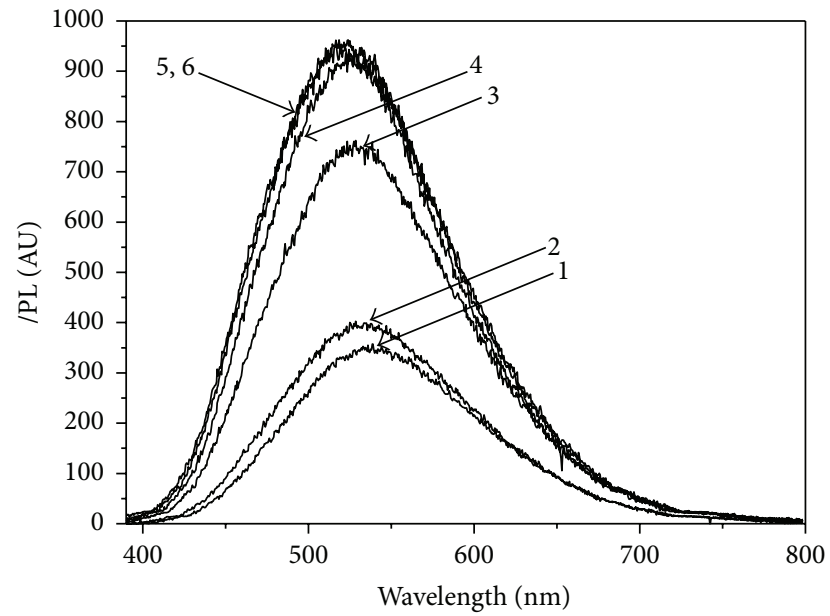

Figure 10: The luminescence spectra of the colloidal solutions of $\mathrm{ZnO}$ nanoparticles without $\mathrm{SiO}_{2}(1)$ and with provisionally adding $0.2 \mathrm{~g}(2), 0.4 \mathrm{~g}(3), 0.6 \mathrm{~g}(4), 0.8 \mathrm{~g}$ (5), and $1 \mathrm{~g}$ of silica (6) to $10 \mathrm{~mL}$ of zinc oxide solution. $[\mathrm{ZnO}]=2 \times 10^{-2} \mathrm{~mol} / \mathrm{L}, l=0.1 \mathrm{~cm}$, and $\lambda_{\text {exc. }}=$ $340 \mathrm{~nm}$.

It was found that the administration of $\mathrm{SiO}_{2}$ nanoparticles during the synthesis of $\mathrm{ZnO}$ nanocrystals leads to a substantial change in the luminescence intensity of nanozinc oxide (Figure 10). The figure shows that the maximum luminescence intensity of $\mathrm{ZnO}$ nanoparticles, into which $\mathrm{SiO}_{2}$ was added during the synthesis, is almost 3 times higher than in the original $\mathrm{ZnO}$ colloid. Thus, it was shown that there is an optimum amount of silica nanoparticles for which $\mathrm{ZnO}$ luminescence intensity reaches its maximum (Figure 11). Further increase in amount of $\mathrm{SiO}_{2}$ added during the synthesis of $\mathrm{ZnO}$ nanoparticles, over the range tested, does not lead to significant changes in luminescence of $\mathrm{ZnO}$ nanoparticles.

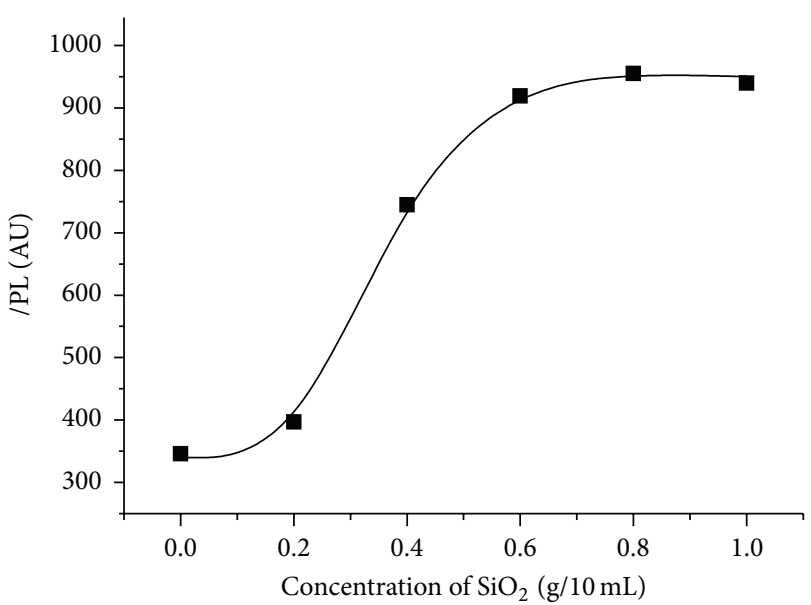

FIGURE 11: The dependence of the maximum of luminescence intensity of $\mathrm{ZnO}$ nanoparticles on the amount of silica added during the synthesis. $[\mathrm{ZnO}]=2 \times 10^{-2} \mathrm{~mol} / \mathrm{L}$.

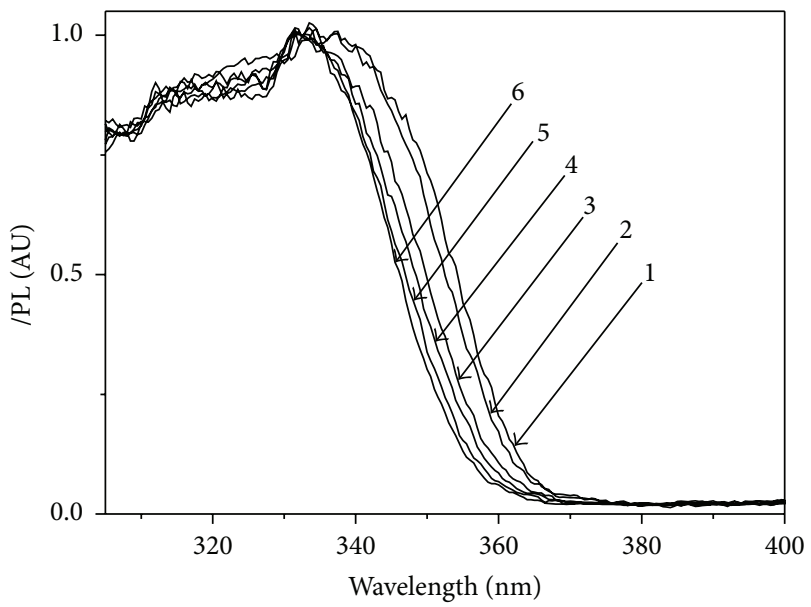

FIgURE 12: Normalized luminescence excitation spectra of the colloidal solutions of $\mathrm{ZnO}$ nanoparticles without $\mathrm{SiO}_{2}(1)$ and with provisionally adding $0.2 \mathrm{~g}(2), 0.4 \mathrm{~g}(3), 0.6 \mathrm{~g}(4), 0.8 \mathrm{~g}(5)$, and $1 \mathrm{~g}$ of silica (6) to $10 \mathrm{~mL}$ of zinc oxide solution. $[\mathrm{ZnO}]=2 \times 10^{-2} \mathrm{~mol} / \mathrm{L}$, $l=0.1 \mathrm{~cm}$, and $\lambda_{\text {emission }}=540 \mathrm{~nm}$.

This substantial increase in luminescence intensity of $\mathrm{ZnO}$ nanocrystals when administering $\mathrm{SiO}_{2}$ during the synthesis, unlike the administering after the synthesis, may be due to a more uniform distribution of $\mathrm{ZnO}$ nanocrystals on the surface of $\mathrm{SiO}_{2}$, as well as minimization of the probability of silica aggregates deposition on the surface of zinc oxide nanoparticles, which is possible when adding $\mathrm{SiO}_{2}$ to the prepared phosphor nanocrystals. Uniform distribution of $\mathrm{ZnO}$ nanocrystals on the surface of silica decreases the probability of separation of the photogenerated charge between the nanoparticles of various sizes, which was discussed in detail above, and as a consequence, it leads to a significant increase in luminescence intensity.

It should be noted that in the absorption spectra (Figure 9) and luminescence excitation spectra (Figure 12) show only minor changes of position of the long-wave edge of 


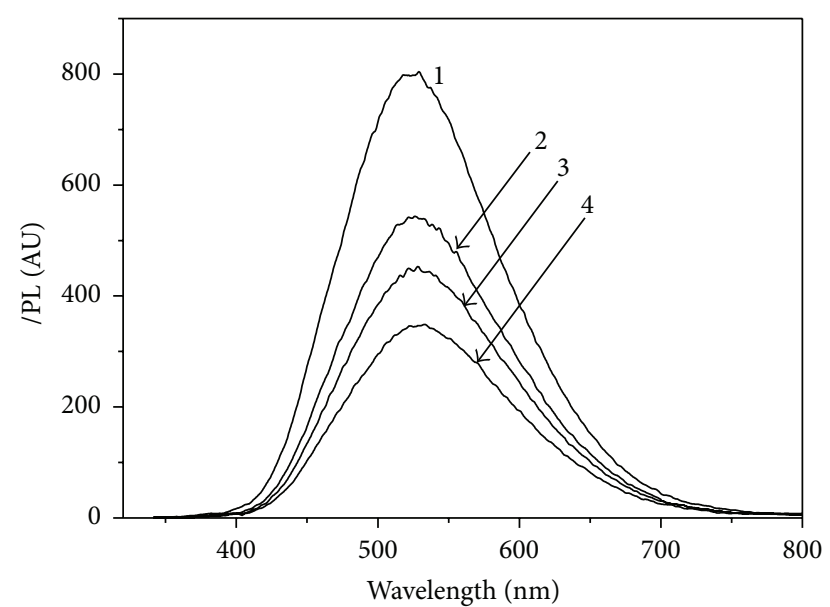

FIGURE 13: The luminescence spectra of $\mathrm{ZnO}$ nanoparticles prepared in the presence of $1 \mathrm{~g}$ of silica per $10 \mathrm{~mL}$ of zinc oxide solution, redispersed in ethanol (1), dimethyl sulfoxide (2), dimethylformamide (3), and ethylene glycol (4). [ZnO] $=2 \times 10^{-2} \mathrm{~mol} / \mathrm{L}$.

the fundamental band of $\mathrm{ZnO}$, when administering silicon oxide during the synthesis of nanoparticles, which indicates its weak influence on the size of the semiconductor.

It was also found that, unlike the individual colloid of zinc oxide, which after removal of the solvent loses its luminescent properties, the material obtained in the study retains its luminescent properties after drying. The powdered $\mathrm{ZnO} / \mathrm{SiO}_{2}$ may subsequently be redispersed either in the source ethanol or in other solvents with retention of high luminescence intensity (Figure 13).

\section{Conclusions}

In this research, it is shown that the dispersion of silica in a solution of zinc oxide nanoparticles allows increasing the luminescence intensity of $\mathrm{ZnO}$ nanoparticles by about $30 \%$. It is found that addition of silica nanocrystals to the source solutions during the synthesis of $\mathrm{ZnO}$ nanoparticles allows increasing their luminescence intensity about 3 times. Thus, the quantum yield of luminescence of $\mathrm{ZnO} / \mathrm{SiO}_{2}$ composites, calculated using a solid anthracene as a standard, is more than $30 \%$. It is suggested that such an impact of silica is caused by the distribution of $\mathrm{ZnO}$ nanocrystals on the surface of silica, which reduces the probability of separation of photogenerated charges between the zinc oxide nanoparticles of different sizes, and as a consequence, there is a significant increase of the luminescence intensity of $\mathrm{ZnO}$ nanoparticles. Enhancement of luminescent properties of $\mathrm{ZnO}$ nanoparticles makes them promising for the use in a variety of devices, such as optical screens, luminescent markers for medical applications, antibacterial coatings for application in food and packaging industry, luminescent solar concentrators, luminescent inks for security printing, and printed smart food packaging. $\mathrm{ZnO}$ nanoparticles with enhanced luminescent properties can be deposited onto surfaces by printing techniques, which opens the possibility of low-cost production of innovative products on an industrial scale.

\section{Competing Interests}

The authors declare that they have no competing interests.

\section{Acknowledgments}

Publications are based on the research provided by the grant support of the State Fund for Fundamental Research (Project N F64/36-2015 from 27.10.15). The research was supported by the Ministry of Education and Science of Ukraine (Project N 2873p).

\section{References}

[1] A. L. Stroyuk, A. I. Kryukov, S. Y. Kuchmii, and V. D. Pokhodenko, "Quantum size effects in the photonics of semiconductor nanoparticles," Theoretical and Experimental Chemistry, vol. 41, no. 2, pp. 67-91, 2005.

[2] H.-M. Xiong, Y. Xu, Q.-G. Ren, and Y.-Y. Xia, "Stable aqueous ZnO@polymer core-shell nanoparticles with tunable photoluminescence and their application in cell imaging," Journal of the American Chemical Society, vol. 130, no. 24, pp. 7522-7523, 2008.

[3] H.-P. Wang, H. Jiang, and X.-M. Wang, "Construction of strong alkaline microcavities for facile synthesis of fluorescencetunable $\mathrm{ZnO}$ quantum dots," Chemical Communications, vol. 46, no. 37, pp. 6900-6902, 2010.

[4] H.-Q. Shi, W.-N. Li, L.-W. Sun, Y. Liu, H.-M. Xiao, and S.-Y. $\mathrm{Fu}$, "Synthesis of silane surface modified $\mathrm{ZnO}$ quantum dots with ultrastable, strong and tunable luminescence," Chemical Communications, vol. 47, no. 43, pp. 11921-11923, 2011.

[5] S. Panigrahi, A. Bera, and D. Basak, "Ordered dispersion of $\mathrm{ZnO}$ quantum dots in $\mathrm{SiO}_{2}$ matrix and its strong emission properties," Journal of Colloid and Interface Science, vol. 353, no. 1, pp. 30-38, 2011.

[6] Z. Fu, B. Yang, L. Li, W. Dong, C. Jia, and W. Wu, "An intense ultraviolet photoluminescence in sol-gel $\mathrm{ZnO}-\mathrm{SiO}_{2}$ nanocomposites," Journal of Physics: Condensed Matter, vol. 15, no. 17, pp. 2867-2873, 2003.

[7] H. He, Y. Wang, and Y. Zou, "Photoluminescence property of $\mathrm{ZnO}-\mathrm{SiO}_{2}$ composites synthesized by sol-gel method," Journal of Physics D: Applied Physics, vol. 36, no. 23, pp. 2972-2975, 2003.

[8] C. M. Mo, Y. H. Li, Y. S. Liu, Y. Zhang, and L. D. Zhang, "Enhancement effect of photoluminescence in assemblies of nano-ZnO particles/silica aerogels," Journal of Applied Physics, vol. 83, no. 8, pp. 4389-4391, 1998.

[9] Y. V. Panasiuk, O. E. Raevskaya, O. L. Stroyuk et al., "Colloidal $\mathrm{ZnO}$ nanocrystals in dimethylsulfoxide: a new synthesis, optical, photo- and electroluminescent properties," Nanotechnology, vol. 25, no. 7, Article ID 075601, 2014.

[10] N. Hagura, T. Takeuchi, S. Takayama, F. Iskandar, and K. Okuyama, "Enhanced photoluminescence of $\mathrm{ZnO}-\mathrm{SiO}_{2}$ nanocomposite particles and the analyses of structure and composition," Journal of Luminescence, vol. 131, no. 1, pp. 138146, 2011

[11] B. Cale, Lab Equipment of Organic Chemistry, Mir, Moscow, Russia, 1966 (Russian). 
[12] D. W. Bahnemann, C. Kormann, and M. R. Hoffmann, "Preparation and characterization of quantum size zinc oxide: a detailed spectroscopic study," Journal of Physical Chemistry, vol. 91, no. 14, pp. 3789-3798, 1987.

[13] A. Wood, M. Giersig, M. Hilgendorff, A. Vilas-Campos, L. M. Liz-Marzán, and P. Mulvaney, "Size effects in $\mathrm{ZnO}$ : the cluster to quantum dot transition," Australian Journal of Chemistry, vol. 56, no. 10, pp. 1051-1057, 2003.

[14] O. L. Stroyuk, V. M. Dzhagan, V. V. Shvalagin, and S. Y. Kuchmiy, "Size-dependent optical properties of colloidal $\mathrm{ZnO}$ nanoparticles charged by photoexcitation," The Journal of Physical Chemistry C, vol. 114, no. 1, pp. 220-225, 2010.

[15] V. I. Mikheev, X-Ray Qualifier of Minerals, Hosheoltehyzdat, Moscow, Russia, 1957 (Russian).

[16] E. M. Wong, P. G. Hoertz, C. J. Liang, B.-M. Shi, G. J. Meyer, and P. C. Searson, "Influence of organic capping ligands on the growth kinetics of $\mathrm{ZnO}$ nanoparticles," Langmuir, vol. 17, no. 26, pp. 8362-8367, 2001.

[17] A. I. Kryukov, S. Y. Kuchmii, and V. D. Pokhodenko, "Energetics of electron processes in semiconductor photocatalytic systems," Theoretical and Experimental Chemistry, vol. 36, no. 2, pp. 6381, 2000.

[18] N. S. Pesika, K. J. Stebe, and P. C. Searson, "Relationship between absorbance spectra and particle size distributions for quantumsized nanocrystals," Journal of Physical Chemistry B, vol. 107, no. 38, pp. 10412-10415, 2003. 

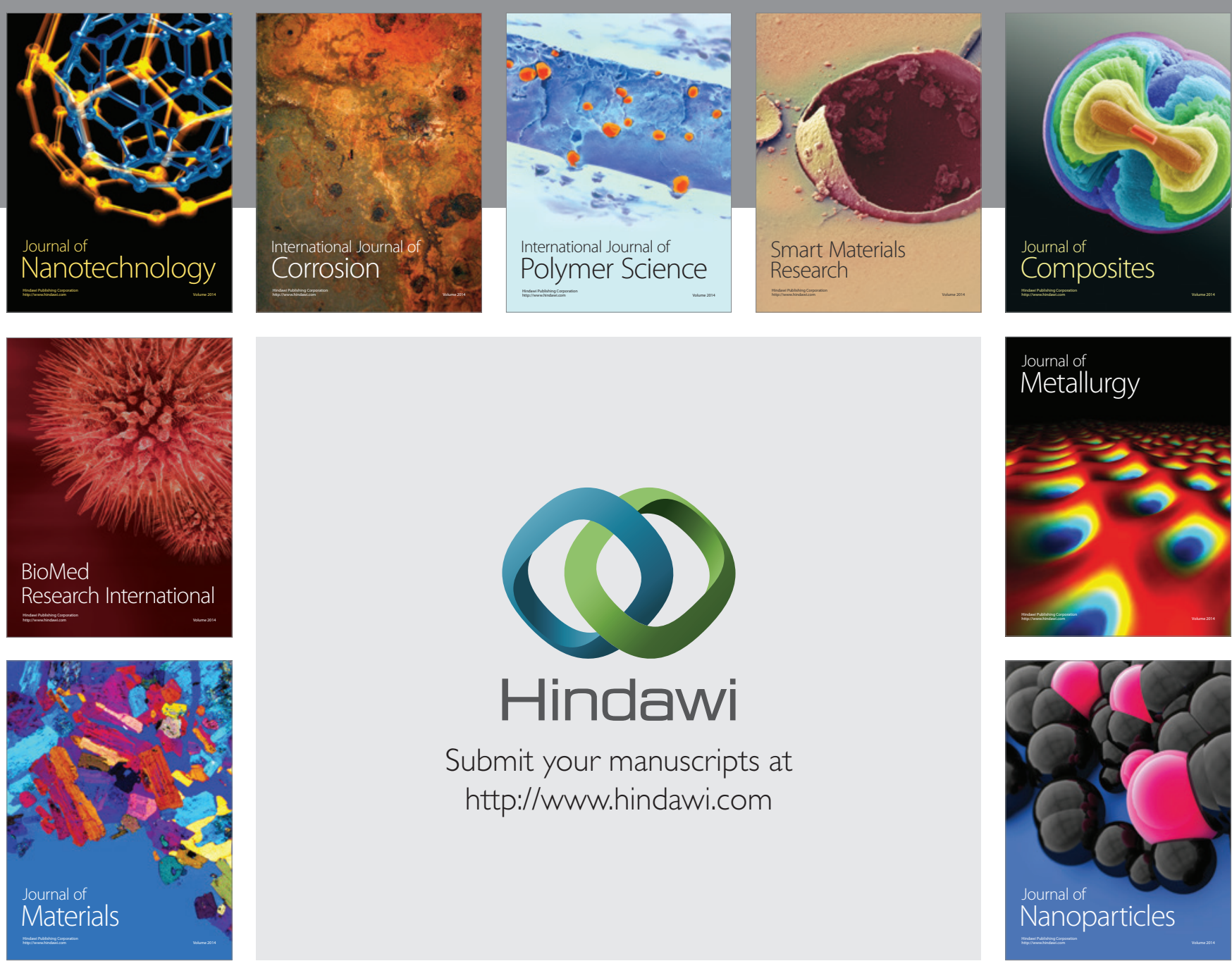

\section{Hindawi}

Submit your manuscripts at

http://www.hindawi.com

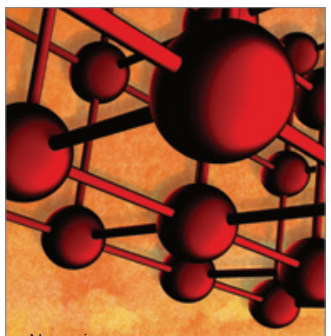

Materials Science and Engineering
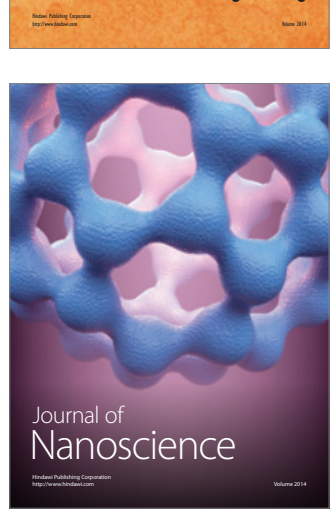
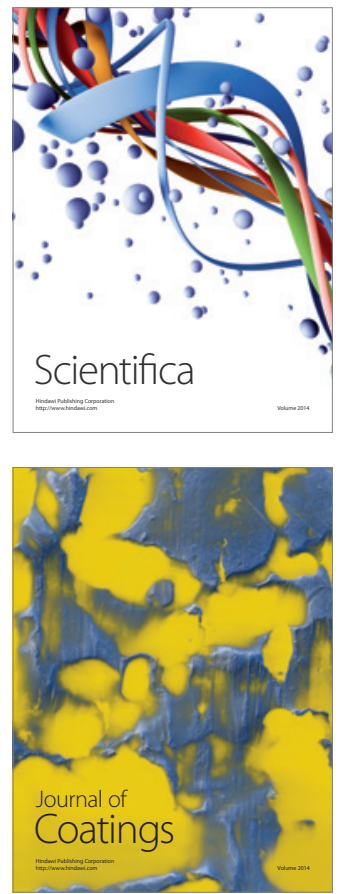
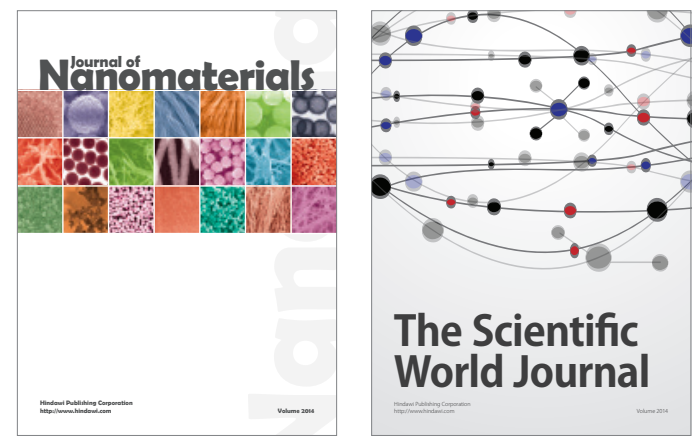

The Scientific World Journal
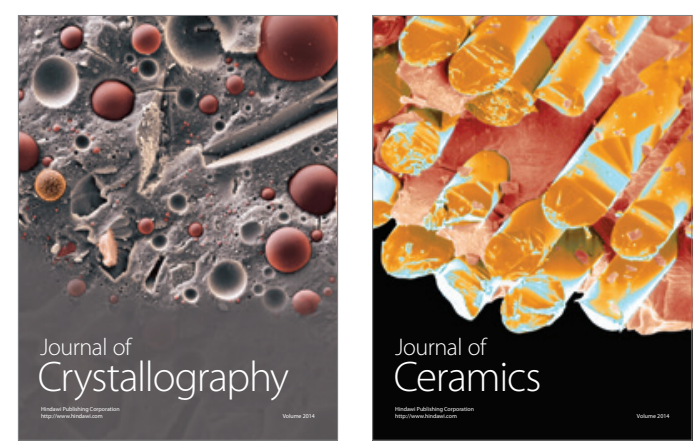
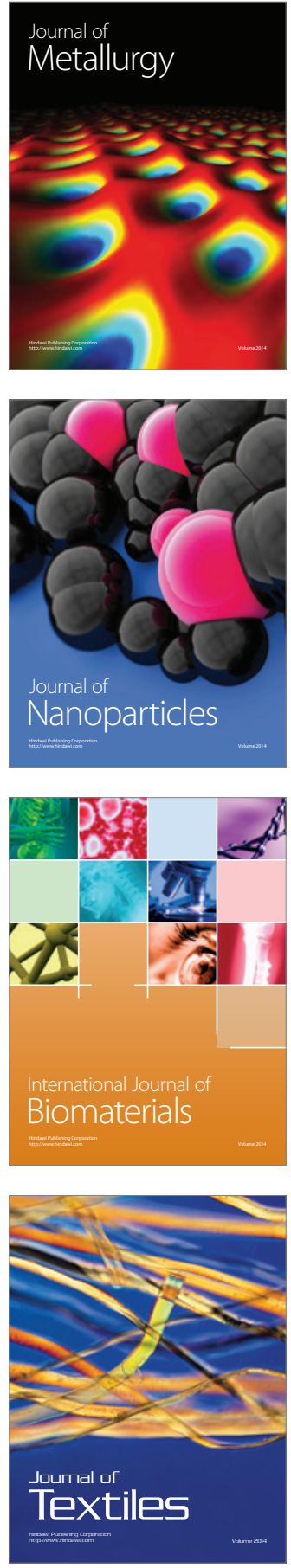